\title{
Transition Delay by Oblique Roughness Elements in a Blasius Boundary-Layer Flow"
}

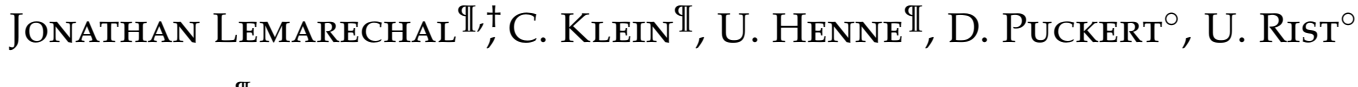 \\ II Deutsches Zentrum für Luft- und Raumfahrt e.V. (DLR), \\ Bunsenstraße 10, D-37073 Göttingen, Germany, jonathan.lemarechal@dlr.de \\ ○ Institut für Aerodynamik und Gasdynamik, Universität Stuttgart \\ Pfaffenwaldring 21, D-70569 Stuttgart, Germany
}

\begin{abstract}
A passive device to delay the laminar-turbulent transition in a Blasius boundary-layer flow is tested at low Reynolds numbers in the laminar water channel of the Institute of Aerodynamics and Gas Dynamics (University of Stuttgart). The passive device consists of oblique surface roughness elements lined up perpendicular to the flow. Each element has the shape of a cuboid, is oriented $45^{\circ}$ to the direction of the oncoming flow, and generates a non-symmetric vortex system. From this vortex system only one vortex per roughness element persists and a co-rotating vortex structure remains after 50 roughness heights downstream of the elements. These vortices generate high and low speed streaks inside of the boundary layer through the lift-up effect. The streaks alter the two-dimensional (2D) boundary layer into a three-dimensional (3D) one. For correctly placed and sized roughness elements the $2 D$ Tollmien-Schlichting wave is altered into a $3 D$ less amplified wave and the transition occurs further downstream. The flow is investigated by means of surface visualizations with the Temperature-Sensitive Paint (TSP) method and velocity measurements with hot-film anemometry. With the TSP method the laminar-turbulent transition was measured with high spatial and temporal resolution. The changes of transition location and changes of transition mechanism are well captured.
\end{abstract}

\section{INTRODUCTION}

The friction drag is the dominating source for drag of streamlined bodies, e.g. wings of aircraft (Schrauf 2005). That is why much effort is made to reduce the viscous drag by increasing the fraction of laminar flow. Reduced friction drag directly leads to energy savings and reduced fuel consumption. In this context several active and passive methods have been developed and tested to increase the portion of laminar flow under different conditions (Reneaux, 2004). For the Blasius boundary-layer flow on a flat plate it is well known that the laminar-turbulent transition is caused by growing two-dimensional Tollmien-Schlichting waves. One possibility to delay the laminar-turbulent transition is to deform these Tollmien-Schlichting waves by introducing alternating regions of increased and decreased flow speed, i.e. high and low speed streaks, respectively, into the boundary layer. If the amplitude and spacing of the streaks is correctly set, then the most unstable two-dimensional Tollmien-Schlichting

\footnotetext{
${ }^{*}$ Citation and credit: Lemarechal, J., Klein, C., Henne, U., Puckert, D.P., Rist, U.: Transition Delay by Oblique Roughness Elements in a Blasius Boundary-Layer Flow. In 2018 AIAA Aerospace Sciences Meeting, 8 - 12 January 2018, Kissimmee, USA. AIAA 20181057 doi:10.2514/6.2018-1057

+Corresponding author: jonathan.lemarechal@dlr.de
}

waves are deformed into three-dimensional TollmienSchlichting waves at a reduced growth rate. This was shown numerically by Cossu and Brandt (2004). In experiments different methods to generate streaks have been tested and all methods have in common that they use the lift-up effect (Landahl, 1980) to generate the necessary streaks of proper amplitude. A streamwise vortex inside of the boundary layer lifts low momentum fluid away from the wall on its one side and pushes high momentum fluid closer to the wall on its other side. The following methods to insert the initial streamwise vortices have been tested to date: discrete surface roughness elements, wavy surface (Downs III and Fransson, 2014), and free-stream turbulence(Fransson et al. 2005b). For the discrete surface roughness elements, which are directly related to the setup of the current test, two different shapes of roughness elements lined up perpendicular to the oncoming flow have been published: Cylindrical roughness elements (Fransson et al. 2005a) and miniature vortex generators(Shahinfar et al., 2012). Both types of surface roughness elements produce pairs of streamwise counter-rotating vortices.

In the following we present an experimental investigation on cuboid roughness elements, which have the same orientation, see Figure 1. The numerical design 
work for the oblique surface roughness was done by Groskopf et al. (2011). At first, each roughness element generates multiple vortices of different strength but only the leading edge main vortex persists. So the identical orientation of all roughness elements generates only co-rotating vortices, which distinguishes the present experiment from previous ones. Groskopf et al. 2011) proposed that the cuboid is supposed to be lying on its largest face, but in the present investigation the cuboid is standing on its long and narrow face as shown in Figure 1. Furthermore, results for off-design conditions are presented.

Investigating this problem in the laminar water channel of the Institute of Aerodynamics and Gas Dynamics is very beneficial because the flow speed is very low and the boundary layer thickness is very large, yet still moderate Reynolds numbers can be reached. The laminar-turbulent transition extends over a long streamwise distance, which makes measurements to determine a transition location very challenging. Therefore, spatial measurement techniques like the thermographic measurement techniques (Fey et al. 2007) are qualified for this application. Performing the measurement in a water facility limits the possibility due to the absorption of light by water to the Temperature-Sensitive Paint (TSP) method and excludes the Infrared technique.

TSP is a possibility to measure two-dimensional surface temperature maps (Liu and Sullivan, 2005). Molecules with the characteristic of a temperaturedependent fluorescence or phosphorescence are embedded in an appropriate surface coating. To perform a surface temperature measurement these molecules are excited by light of appropriate wavelength and the emitted fluorescence or phosphorescence light is measured with a camera, for example.

Temperature-sensitive paint can be used as a thermographic measurement technique (called TSP method) to visualize and also to quantitatively measure some aspects of laminar-turbulent transition, vortex footprints, streaks, and laminar separation bubbles (Liu and Sullivan, 2005; Fey and Egami, 2007). The measurement principle of the TSP method is based on the Reynolds-Colburn analogy, i.e. differences in temperature distribution are linked to variations in local skin friction for a constant heat flux, fluid temperature, and flow speed. The TSP method fulfills the requirements for the measurements concerning acquisition frequency and size of measurement area in the laminar water channel. In this laminar-turbulent transition was measured with such a high degree of temporal and spatial resolution with the TSP method for the first time.

\section{EXPERIMENTAL SETUP}

The test was conducted in the laminar water channel "Laminarwasserkanal" of the Institute of Aerodynamics and Gas Dynamics (University of Stuttgart). The facilities characteristics are very suitable for tests investigating the laminar-turbulent transition of a Blasius boundary-layer flow - here on a flat plate of $8 \mathrm{~m}$ length and $12 \mathrm{~m}$ width with an elliptic leading edge. Velocities up to $0.2 \mathrm{~m} / \mathrm{s}$ can be reached with a low turbulence level of $\mathrm{Tu}=0.05 \%$ in the range of $0.1 \mathrm{~Hz}$ to $10 \mathrm{~Hz}$ (Strunz, 1987). On top of the flat plate the water level measures $0.15 \mathrm{~m}$, which is sufficiently high to have no blockage effects and a free water surface without disturbances with the installations of the present experiment. Throughout the test campaign the velocity was fixed at $0.12 \mathrm{~m} / \mathrm{s}$ with a variation of less than $1 \%$. The setup is shown in 2 and consisted of three installations, i.e. the array of roughness elements, a controlled disturbance generation with a vibrating ribbon and a movable device to perform TSP measurements in the laminar water channel which is referred to as TSP element.

\subsection{TSP Setup}

A TSP setup consists of three components, i.e. the temperature-sensitive paint itself, a possibility to excite the TSP molecules, and a device to record the emission of the TSP. The temperature-sensitive dye molecules used in this experiment are explained in detail by Ondrus et al. (2015) and have already been used in previous experiments in wind tunnels (Ondrus et al. 2015) and water facilities (Fey et al. 2013). For the used TSP formulation near UV light is required for excitation and red light is emitted from the TSP. The absorption spectrum of water shows that both utilized wave lengths have a sufficiently small absorption in water (Wang, 2008) to enable TSP measurements.

The high flow quality of the laminar water channel results in an undisturbed free water surface, which permits to take the TSP images through the free water surface. The distance between flat plate and the ceiling is limiting for the optical setup. Thus a three camera setup was used. Three scientific black and white charge-coupled device (CCD) cameras with low noise were used to acquire the TSP images. In this setup shading effects, which occur when the entire 
CCD chip is used, were avoided by using just the center region of the CCD chip. This improved the results especially for the calculation of the standard deviation of each pixel. The combination of optical lenses and camera settings results in a resolution of 4.4 pixels per millimeter. By maximizing the distance between the cameras and the flat plate surface the TSP element could not be placed symmetrically to the channel walls. In Figure 1 the right-handed coordinate system used throughout this paper is defined. The $x y$-plane is in the center line of the TSP coated area. Four light-emitting diodes (LEDs) with a peak emission at $405 \mathrm{~nm}$ (HARDsoft IL-105/6X Illuminator UV) were used to excite the temperature-sensitive dye molecules. Appropriate optical filters were used in front of the cameras and LEDs to avoid reflections.

\subsection{Roughness Elements}

In the current work cuboid roughness elements of width $w$, length $l$, and height $k$ have been tested as illustrated in Figure 1 . These roughness elements are lined up in $z$ direction in order to introduce high and low speed streaks into the Blasius boundary layer. The proportions of the cuboid and their orientation of $\varphi=45^{\circ}$ to the mean flow direction were designed by Groskopf et al. (2011). The width of the elements is wider than specified by Groskopf et al. (2011) due to manufacturing reasons. The elements are made from brass; therefore, they do not require any additional fixing to stay in place because of their own weight at the low flow speeds in the laminar water channel. The height of the elements in relation to the boundary layer at the roughness location $x_{k}$ is based on Fransson et al. (2005a). In their experiment the cylindrical roughness elements generate streaks of appropriate strength for a cylinder height of $k / \delta=2.6-2.8$ with the boundary layer parameter $\delta=\left(x v / U_{\infty}\right)^{0.5}$. In the experiment of Fransson et al. (2005a) spanwise spacing was $\Delta z / k=$ 4.2. This was increased significantly in the present experiment to $\Delta z / k=11$. In Table 1 the dimensions of the investigated roughness elements and the used roughness locations are listed. The roughness location $x_{k}$ was varied between $0.150 \mathrm{~m}$ and $0.350 \mathrm{~m}$ to vary the amplitude of the generated streaks close to the transition location of the reference conditions. In order to further quantify the effect of the roughness elements on the boundary layer also the roughness Reynolds number $\operatorname{Re}_{k k}=u_{(y=k)} k / v$ is determined. $u_{(y=k)}$ is the velocity at the location of the roughness elements at the top of the roughness element.

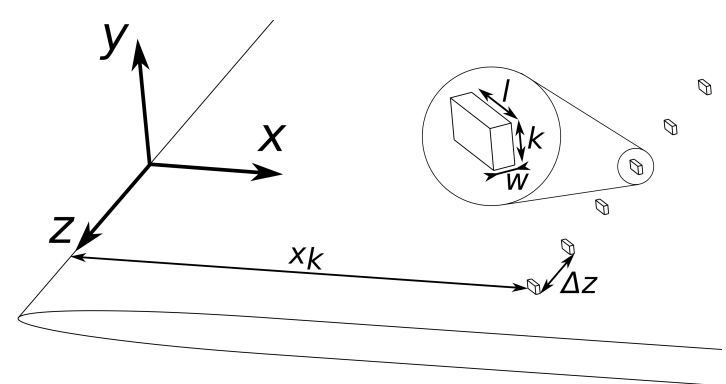

Figure 1: Schematic illustration of the roughness elements dimensions, their orientation, location, and spacing.

Table 1: Summary of the measurement parameters and dimensions of the roughness elements of the presented test cases.

\begin{tabular}{cccccc}
\hline Parameter & $(\mathrm{I})$ & $(\mathrm{II})$ & $(\mathrm{III})$ & $(\mathrm{IV})$ & $(\mathrm{V})$ \\
\hline$k[\mathrm{~mm}]$ & 6.5 & - & 3.5 & 3.5 & 3.5 \\
$w[\mathrm{~mm}]$ & 3.4 & - & 2.0 & 2.0 & 2.0 \\
$l[\mathrm{~mm}]$ & 13.3 & - & 7.2 & 7.2 & 7.2 \\
$x_{k}[\mathrm{~m}]$ & 3.7 & - & 0.225 & 0.275 & 0.325 \\
$\Delta z[\mathrm{~mm}]$ & 71.5 & - & 38.5 & 38.5 & 38.5 \\
$\varphi\left[^{\circ}\right]$ & 45 & - & 45 & 45 & 45 \\
$F[-]$ & - & 90 & 90 & 90 & 90 \\
$k / \delta$ & 1.15 & - & 2.51 & 2.27 & 2.09 \\
$\operatorname{Re}_{k k}$ & 276 & - & 283 & 263 & 254 \\
\hline
\end{tabular}

\subsection{Controlled Disturbance Generation}

A vibrating ribbon is used to introduce a controlled disturbance into the Blasius boundary-layer flow. The vibrating ribbon is located at $x=1.65 \mathrm{~m}$, which corresponds to Branch I of the stability diagram. A thin wire (diameter of $0.1 \mathrm{~mm}$ ) oscillates at an amplitude of $0.25 \mathrm{~mm}$, has an offset of $y=5 \mathrm{~mm}$, and a dimensionless frequency $F=2 \pi f v / U_{\infty}^{2} \cdot 10^{6} \approx 90$. Underneath the vibrating ribbon a series of spacers $(55 \mathrm{~mm}$ $\times 29 \mathrm{~mm} \times 1 \mathrm{~mm}$ ) were aligned with a distance of $55 \mathrm{~mm}$. A similar setup with wider spacers and a different dimensionless frequency $F$ was tested in the laminar water channel before (Kruse and Wagner 1998). The spacers cause a fixed three-dimensional deformation of the boundary layer, which results in a K-type transition(Kruse and Wagner. 1998; Klebanoff et al. 1962). This spanwise fixing of the transition location reduces the demand for the spanwise extent of the TSP coated area and enables to compare the surface visualizations with TSP and velocity measurements with hot-film anemometry, which could not be performed simultaneously. 


\subsection{TSP Element}

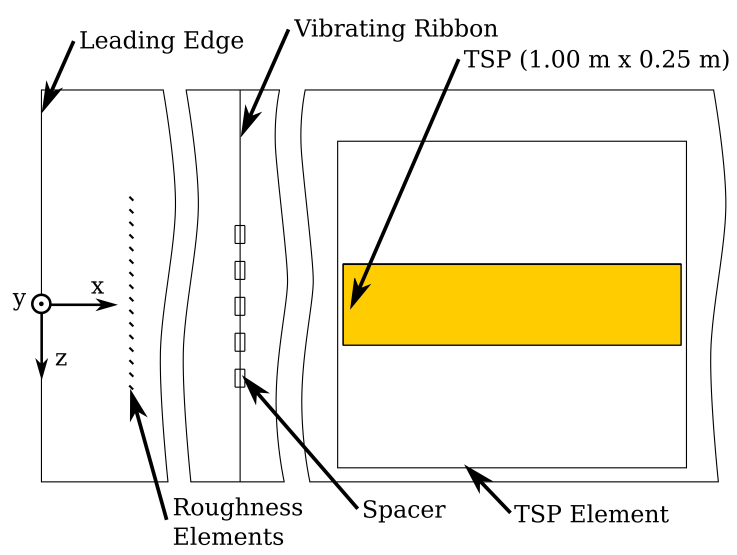

Figure 2: Top view of the experimental setup.

To enable TSP measurements in the laminar water channel a flexible, i.e. moveable, solution for creating a temperature difference between surface and fluid that can also serve as substrate for the TSP coating had to be designed. The final design of the TSP element consists of an aluminum plate $(1.07 \mathrm{~m} \times 100 \mathrm{~m}$ $\times 0.001 \mathrm{~m}$ ) onto which several layers of glass fiber reinforced plastic have been laminated. One single layer of carbon fiber and one single layer of aluminum foil are embedded inside of the insulating glass fiber reinforced plastic. The metallic substrate is used to keep the TSP element in place and ensure that the TSP element keeps its flatness. The single layer of carbon fiber is used as a resistive heating layer, as it was proposed by Fey and Egami (2007) and already used by Petzold and Radespiel (2015) in a low speed wind tunnel test. With the integrated aluminum foil minor temperature homogeneities are evened out. Finally, the active layer containing the temperature-sensitive dye molecules was spray coated on an area of $1.00 \mathrm{~m} \times 0.25 \mathrm{~m}(20 \%$ of test section width) and the remaining surface was coated matt black. In order to limit the amount of thermal energy introduced into the water channel flow during a TSP measurement the spanwise extent of the heated area was limited to a width of $0.35 \mathrm{~m}$, which is wider than the TSP area to avoid possible edge heating temperature inhomogeneity close to the electrical contact of the heating. Possible non-homogeneous heat flux would cause severe deterioration of TSP results. A large heated surface or a large heat flux will cause buoyancy forces and thermally induced turbulence. These effects have to be avoided to perform non-intrusive surface visualizations. The heating is split in two parts with a gap of $20 \mathrm{~mm}$. The overall thickness is $d=1.9 \mathrm{~mm}$, which results in $d / \delta * \leq 0.21$ for the least favorable flow conditions and chosen locations of the TSP element in the current test. The leading edge of the TSP element is designed as a sequence of two forward-facing steps with a height of $1 \mathrm{~mm}$ for the first step and a height of $0.9 \mathrm{~mm}$ for the second one. Between the two steps there is a small plateau of $70 \mathrm{~mm}$ length. A previous design of the TSP element was already used by Lemarechal et al. (2018).

\section{Data Acquisition and Data Evaluation}

The Temperature-Sensitive Paint method is applied using the intensity based method (Liu and Sullivan 2005). The TSP molecules were excited continuously with the LEDs and images were recorded. The images were acquired with a frequency of $10 \mathrm{~Hz}$ and an exposure time of $4.5 \mathrm{~ms}$. This means that the images were acquired at 50 times the disturbance frequency introduced by the vibrating ribbon. The TSP data acquisition strictly followed a fixed sequence for each measurement point during which the water channel was permanently running:

- Ten dark images, i.e. without LED light, are acquired to measure the background light.

- The LEDs are switched on in continuous mode to acquire 100 reference images, with the surface temperature of the TSP element and the fluid temperature being in equilibrium. Images acquired under this condition are called reference images.

- The heating is switched on at a constant electrical power. The electrical heating was set to an electrical input power of $120 \mathrm{~W}$, which results in an over temperature of approximately $2 \mathrm{~K}$ equal to a temperature ratio of $T_{w} / T_{a w}=1.007$ in the laminar region.

- When the surface temperature is constant the LEDs are switched on again and 900 images are acquired now at a constant heat flux between surface and fluid. These images are called run images.

The in-house developed software nToPas (Sachs, 2007) was used for the evaluation of the data. At first the data are prepared in the image plane: dark, reference, and run images are averaged and afterwards the averaged dark image is subtracted from the averaged run and reference images. Four different ways to present the data are prepared: averaged result, time 
series, standard deviation, and amplitude spectrum calculated by Fourier analysis.

In the instantaneous results of the time series data a horizontal regular stripe pattern unregularly appears besides the random noise (Figure 6 a)). To remove these patterns and to increase the signal-to-noise ratio the singular-value decomposition (SVD) is used to filter the data. SVD is used for image filtering and denoising (Konstantinides et al., 1997) and it is very suitable for the acquired data because the filter is edge preserving and does not require previous information about the image or the noise. Furthermore, it has been used to increase the signal-to-noisy ratio of pressuresensitive paint data by Pastuhoff et al. (2013) and it is used in flow analysis (Taira et al. 2017). The SVD factorizes a $m \times n$ matrix $\mathbf{A}$ into a unitary matrix $\mathbf{U}$ containing the left singular vectors $\mathbf{u}_{\mathbf{i}}$, a diagonal matrix $\Sigma$ containing the singular values $\sigma_{i}$, and a unitary matrix $\mathbf{V}^{\mathbf{T}}$ containing the right singular vectors $\mathbf{v}_{\mathbf{i}}^{\mathbf{T}}$ :

$$
A=U \Sigma V^{T}=\sum_{i=1}^{N} \sigma_{i} u_{i} v_{i}^{T}
$$

The time series results, which are handled as a three-dimensional matrix $(x \times y \times t)$ until the SVD is applied, are rearranged into a two-dimensional matrix $(x \cdot y \times t)$ in which the spatial component is reshaped into a one-dimensional vector. The vector $\boldsymbol{u}_{i}$ contains spatial and the vector $v_{i}^{T}$ temporal components of the factorization. $u_{1}$ is the average spatial component and all further components $u-i$ contain the higher order components. The singular values are the square root of the eigenvalues $\sigma_{i}$ in descending order and represent the energy of each component.

In the present experiment a wide range of frequencies occur, which is why the criteria to exclude components in the reconstruction had to be chosen more flexible than in Pastuhoff et al. (2013). For the reconstruction of the time signal all components are included until $50 \%$ of the total energy of the fluctuating components is reached, this is shown in Figure 4 a). As expected the first components contain the most energy, which can also be seen for the spatial component $\mathrm{u} 2$ shown in Figure 3, which is already very similar to the averaged result in Figure 6 a). For the shown examples 344 (case (II)) and 350(case (III)) of the 900 components are included. In Figure 4 a) it can be seen that the energy of the excluded components is equal for the two data points. This indicates that they only contain camera noise. Furthermore, all components being dominated by a pattern that is ascribed to the camera read out are manually excluded. In Figure
3 the first 14 spatial components for the reference flow, i.e. case (II), are shown. The spatial components $u_{3}$, $u_{6}, u_{11}$, and $u_{14}$ were excluded from the reconstruction because of the dominating horizontal stripe pattern. In the components $u_{7}$ and $u_{8}$ (Figure 3 ) a significant difference can be seen on the upstream end of the TSP element: The difference of the noise level of this analog-to-digital converter leads to a locally increased energy level of these components.

The global error and pixel error are also calculated as proposed by Pastuhoff et al. (2013) to check the success of the image filtering and to check if all important characteristics are included in the reconstructed image. The pixel error is calculated, if possible, in a laminar region and the global error is calculated over an area of $50 \mathrm{~mm} \times 100 \mathrm{~mm}$, which is in the order of typical structures in this test: footprints of $\lambda$-vortices. A comparison of an unfiltered and SVD filtered instantaneous image is shown in Figure 7. It is visible that the random stripe patterns and the noise are significantly reduced.

The unfiltered time series is used as input for a Fourier analysis. The signal-to-noise ratio of the data in the present measurement is of sufficient quality to directly calculate the amplitude spectrum $|Y|$ for each grid point without quality enhancing methods as presented by Nakakita (2007) for pressure-sensitive paint data. Because of the similarity of SVD method and Fourier analysis it is not necessary to use the filtered data. The Fourier analysis is used to specifically highlight the dimensionless frequency band $F=90 \pm 10$, which is the frequency band centered at the controlled disturbance. If the amplitude in this frequency band is significantly increased in the TSP results, it is expected that the transition start is determined. The results of the reference flow support this assumption: The amplitude of the dimensionless frequency band $F=90 \pm 10$ of the TSP results (Figure 6 has its maximum at $\operatorname{Re}_{x}=4.05 \cdot 10^{5}$ same as the standard deviation of the velocity $u$ (Figure 8).

Velocity measurements were performed with hotfilm anemometry in constant temperature (CTA) mode with a single wire probe (Dantec 55R15) at a temperate ratio of 1.08 . The calibration was performed with the traversing system of the laminar water channel as described by Subasi et al. (2015). The probe was traversed parallel to the TSP element surface at a constant height of $y=7 \mathrm{~mm}$. Therefore, possible disturbances caused by the TSP element are also included in the velocity measurements with the hot-film anemometry. The measurements started at the leading edge of the TSP element and $z=-120 \mathrm{~mm}$ with 


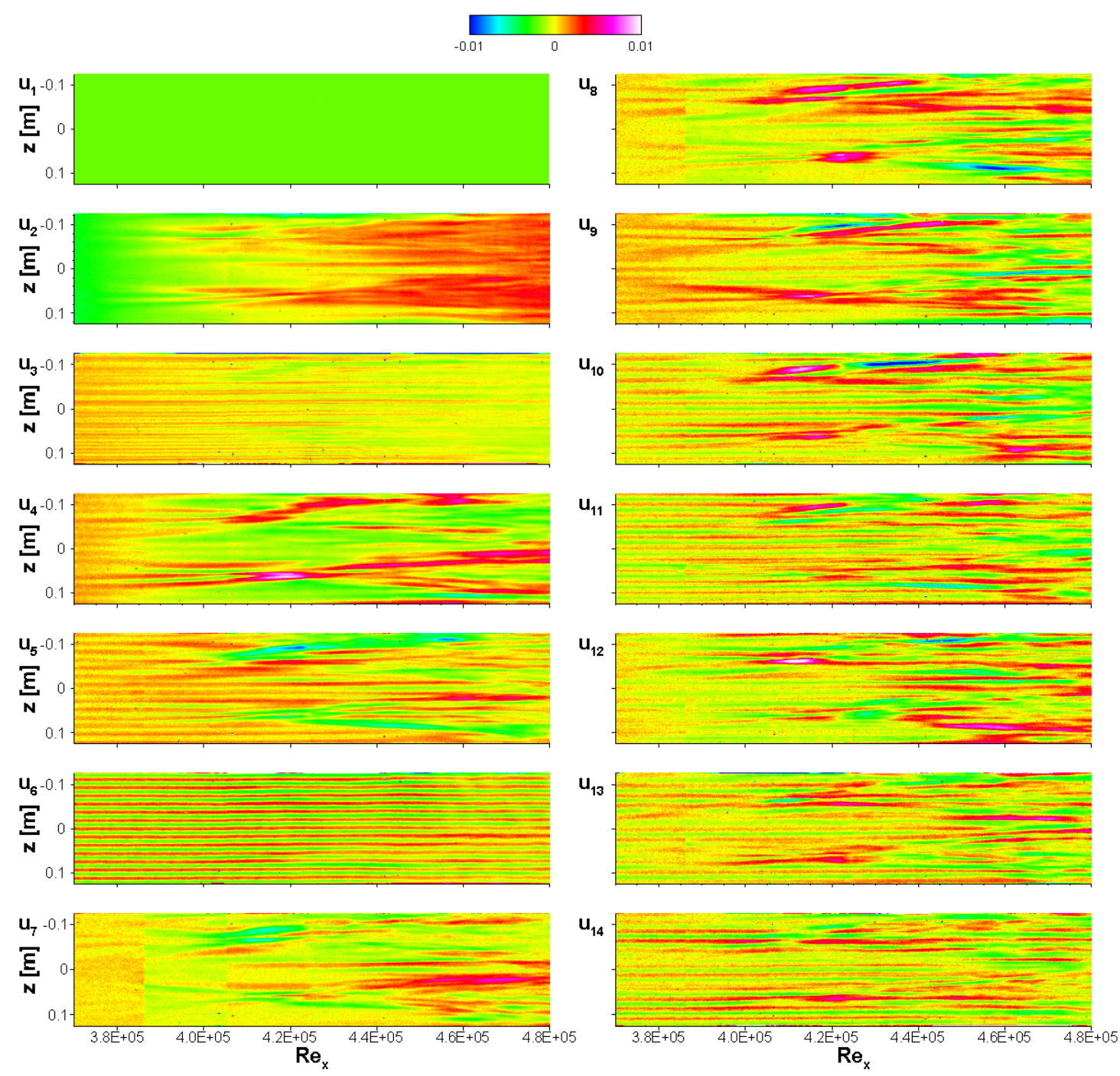

Figure 3: The spatial components $u_{1}$ to $u_{14}$ for the reference flow, i.e. case (II), are shown. The components $u_{3}, u_{6}, u_{11}$, and $u_{14}$ are dominated by disturbances coming from the camera.

a spacing of $\Delta x=25 \mathrm{~mm}$ and $\Delta z=10 \mathrm{~mm}$. At each measurement location the velocity was measured with at a frequency of $100 \mathrm{~Hz}$. The hot-film measurements were performed between two TSP measurements of the same setup without any change of the setup.

\section{EXPERIMENTAL Results}

Results are shown for five different cases as specified in Table 1. Case (I) is a visualization of the close-up range of the roughness elements to compare with numerical results from Groskopf et al. (2011). This visualization is measured with scaled roughness elements (larger elements than in cases (III) to (V)) further downstream on the flat plate. The scaled elements at the downstream location have a roughness Reynolds number $\operatorname{Re}_{k k}$ between case (III) and case (IV). Scaling the elements and placing them further downstream was necessary because the TSP element would generate a non-negligible disturbance close to the leading edge: The forward-facing step at the TSP elements leading edge would be in the order of the displacement thickness of the Blasius boundary layer. Case (II) is the reference flow configuration this means that the controlled disturbance is excited but the oblique roughness elements are not used. For case (III) to (V) the oblique roughness elements are used. Their location is moved consecutively in downstream 
a)

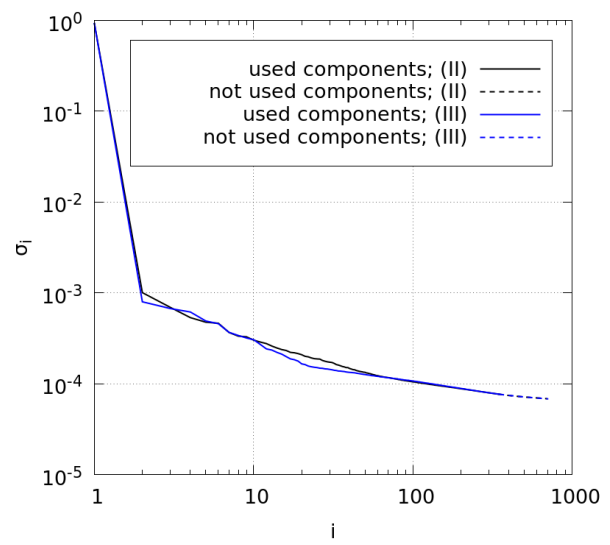

b)

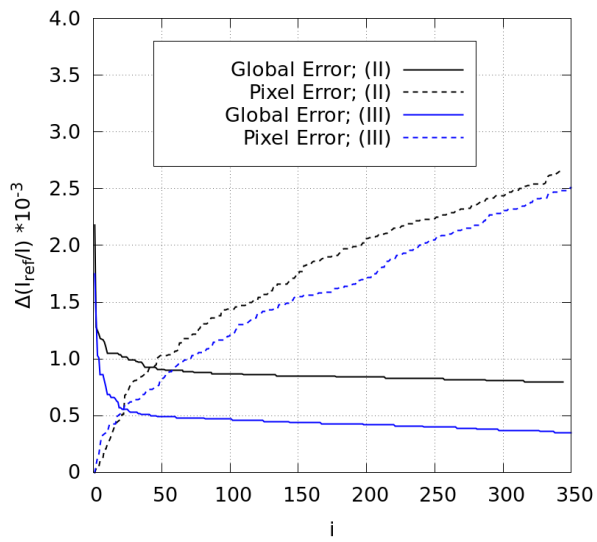

Figure 4: Energy of each component $i$ a) and global and pixel error $b$ ) of each included component $i$ of the reference flow, i.e. case (II), and for oversized roughness elements, i.e. case (III).

direction from case (III) to (V), this means that the strength of the streaks and the roughness Reynolds number $\operatorname{Re}_{k k}$ are reduced. For these test parameters the influence of the generated system of high and low speed streaks on the laminar-turbulent transition is investigated with the disturbance environment of case (II). The cases (III) and (V) are off-design conditions.

The presented average and instantaneous TSP results have to be interpreted in the following manner: locations of low intensity ratio $I_{r e f} / I$, i.e. the temporal average of the acquired intensity values at reference conditions divided by the temporal average of the intensity of the run image, belong to locations of larger absolute local skin friction. This conclusion is valid for the present experiment because the heat flux, the distribution of heat conductivity in the model, and the flow conditions are homogenous and time independent. The cause of increased skin friction has to be deduced from the flow situation and with the help of the standard deviation and the Fourier analysis.

\subsection{Close-up Range of the Roughness Ele- ments}

To visualize the vortex system generated by the oblique roughness elements a new arrangement with larger elements was tested. These elements have a height of $k=6.5 \mathrm{~mm}$ and are directly placed on to the TSP element. Their size and location was set to have roughness Reynolds number of $\operatorname{Re}_{k k}=276$, which is similar to case (III). The footprints of the vortex system can be seen clearly as darker areas in Figure 5 Each roughness element generates three vortices that are sufficiently strong to change the local skin friction enough for the TSP method to clearly visualize them as individual dark shapes. The naming of the vortices follows the convention introduced by Groskopf et al. (2011) but the distinction between inner and main vortex is omitted, because this information is not available from the TSP measurements. One vortex, which is called horseshoe vortex (HV), is bent around the element and reaches no further downstream than five roughness heights. The second visible vortex is the trailing edge vortex (TV). It originates from the trailing edge of the roughness element, is about half as wide as the roughness element, rapidly weakens, and is only visible up to $\left(x-x_{k}\right) / k \approx 30-45$. The vortex inducing the ordered streak system to delay the laminar-turbulent transition is the leading edge vortex (LV). It is visible from $5 k$ on and its strength and width are increasing in flow direction, which is concluded from the decreasing intensity ratio. This vortex is supposedly more stable than the pair of counter-rotating vortices a symmetrically oriented roughness element would generate (Groskopf et al. 2011). Especially, the trailing edge vortex shows a different development in flow direction for the three roughness elements visible in the TSP result. The TV generated by the roughness element at $z / k=11$ is extinct at $30 k$ downstream of the elements leading edge whereas the TV for the roughness element at $z / k=0$ is visible up to $45 k$ downstream of its roughness element. Possible causes are a small deviation in the angle of the elements or a variation in the mean flow field. The comparison of the results with the cold-flow conditions published by Groskopf et al. (2011) is fairly good with a minor deviation for the location at which the end of the TV is detected. This difference is probably caused by the decreasing influence of the vortex on the skin friction or the inverted height to width ratio. Also the spanwise movement of the vortices in positive $\mathrm{z}$ direction is similar for the current experimental results and the numerical results (Groskopf et al. 2011). 


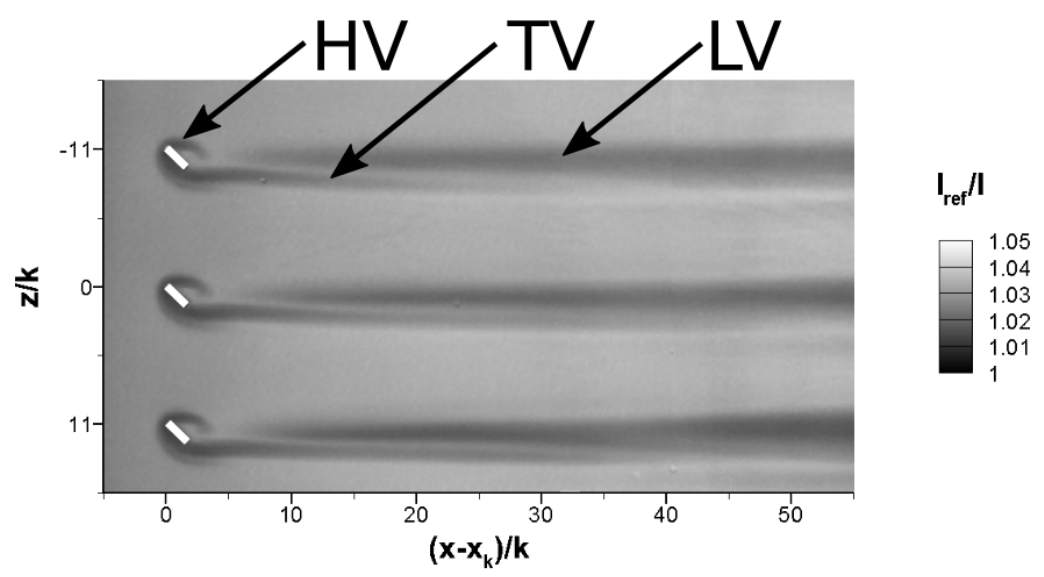

Figure 5: The averaged TSP result of the close-up range of the roughness elements (case (I)) shows the footprints of the vortices: Horseshoe vortex $(H V)$, leading edge vortex $(L V)$, and trailing edge vortex (TV).

\subsection{K-Type Transition Reference Flow}

The reference flow is dominated by appearing structures caused by the previously defined disturbance environment without roughness elements. These conditions lead to the averaged TSP result shown in Figure 6a). Two darker wedge shapes start at $\operatorname{Re}_{x}=4.15 \cdot 10^{5}$ which are assumed to be turbulent wedges caused by the disturbance of the vibrating ribbon and the spacers. Three dark footprints separated by brighter lines lead up to the tip of the turbulent wedge. The spanwise gap between the outer dark lines is approximately $55 \mathrm{~mm}$ and the width of the individual formation is also approximately $55 \mathrm{~mm}$ at the beginning of the TSP measurement area. This formation reflects the dimensions and the spanwise distance of the spacers beneath the vibrating ribbon. The central black strip, which is going straight to the tip of the turbulent wedge, is located downstream of the centerline between two spacer (Kruse and Wagner, 1998). This stationary deformation of the boundary layer results in the formation of $\lambda$ vortices (Hama and Nutant, 1963), which become unstable and develop into turbulent flow. The $\lambda$ vortices are initiated at discrete spanwise location and are lined up (Figure 7), which leads to the conclusion that K-type or fundamental type transition was successfully achieved. In the tip of the turbulent wedge the standard deviation (Figure 6 b)) and also the amplitude spectrum (Figure 6 c)) of the TSP data show their maximum. The amplitude spectrum shows the dimensionless frequency range $F=90 \pm 10$, thus the amplitude of the introduced disturbance is indirectly measured. The increased values in the wedge's tip are good indication that the wedges are turbulent and that they are generated by the disturbance of the vibrating ribbon. The generated $\lambda$ vortices decay further downstream into smaller vortices. These decaying structures still cause significantly larger values in the amplitude spectrum compared to upstream of the wedge's tip. However, the standard deviation in Figure 6 b) clearly highlights only the borders of the wedge as a bright line and thus the boundary between the laminar and the turbulent flow. The upstream end of the increased values of the amplitude spectrum is considered to be the transition location. Inside of the wedge shape the averaged TSP result shows a dark area with $I_{r e f} / I \leq 1.02$, which is assumed to correspond to the increased skin friction of turbulent flow. The transition Reynolds number of the reference flow was reproducible thus the disturbance generated by the vibrating ribbon seems to be very stable.

On the positive $z$-coordinate side of the instantaneous result (Figure 7) three different conditions of the $\lambda$ vortex are visible: initiation, growth and decay. Initially, the footprint of a $\lambda$ vortex is visible in TSP data as a small structure of two dark parallel lines at $\operatorname{Re}_{x}=4.15 \cdot 10^{5}$. An elongated pair of dark parallel lines from $\operatorname{Re}_{x}=4.30 \cdot 10^{5}$ to $\operatorname{Re}_{x}=4.50 \cdot 10^{5}$ shows the next development step: a grown vortex. And finally at $\operatorname{Re}_{x}=4.60 \cdot 10^{5}$ the vortex is decaying into smaller structures. This evolution shows the convective nature of the instability. These events strictly occur regularly with $F=90$ and the size of the two turbulent wedges hardly changes, as it can be seen in the results of the standard deviation, see Figure 6 c). On the negative $z$-coordinate side it is not possible to identify individual stages of the vortex evolution, which is very likely caused by a faster evolution due 

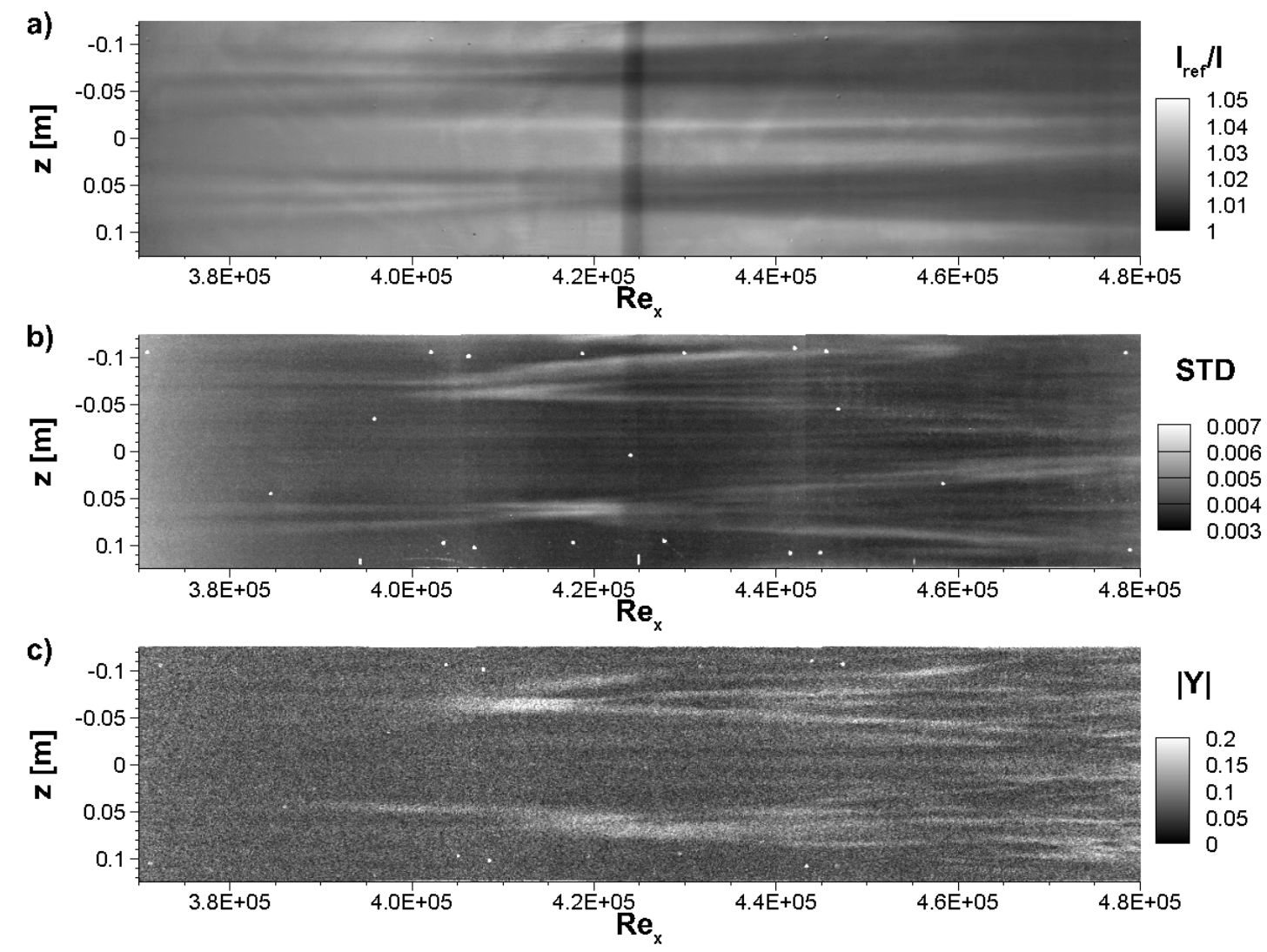

Figure 6: TSP results for the reference flow, i.e. case (II). The averaged result a), the standard deviation b), and the amplitude of the $F=90 \pm 10$ frequency range derived from Fourier analysis c) are shown.

to locally larger disturbance.

The standard deviation also shows vertical lines at $\operatorname{Re}_{x}=3.88 \cdot 10^{5}, 4.06 \cdot 10^{5}, 4.25 \cdot 10^{5}$, and $4.45 \cdot 10^{5}$ which are caused by a small difference of the noise level of each analog-to-digital converter (two analogto-digital converters per camera). A further striking feature is one vertical dark stripe starting at $\operatorname{Re}_{x}=$ $4.25 \cdot 10^{5}$, which is not a flow feature but caused by the gap between the two heating elements. The break in the heating is clearly visible in the average TSP result, c.f. Figure 6a), but it is not visible in the standard deviation, c.f. Figure 6 c), which confirms that the surface temperature of the TSP element had reached a constant condition before the image acquisition of the heated images started.

For the reference case the TSP measurements were accompanied with hot-film measurements. The result of the averaged velocity $u$ is shown in Figure 8 a). Like the averaged TSP results, also in the hot-film measurement results a wedge shaped area of increased flow speed is visible. Already upstream of the wedge shape a non-two-dimensional boundary layer is detected: A structure in flow direction of approximately $50 \mathrm{~mm}$ width and an elevated flow speed leading up to tip of each turbulent wedge is visible. Furthermore, an increased flow speed is visible at the beginning of the TSP element, which is than decelerated between the parallel structures. The standard deviation derived from the velocity measurements is displayed in Figure $8 \mathrm{~b})$. It shows more clearly than the average result two parallel horizontal structures with a distance between their centerlines of $120 \mathrm{~mm}$. Also the spanwise location resembles the one measured with the TSP method. The standard deviation inside of the parallel structures increases at $\operatorname{Re}_{x} \approx 3.80 \cdot 10^{5}$ (Figure 8 b)), which is earlier compared to TSP results where the increase is detected at $\operatorname{Re}_{x} \approx 4.00 \cdot 10^{5}$ (Figure 6 c)). Nevertheless, the maximum standard deviation inside the growing disturbance is for both techniques at a similar location for each vortex, i.e. $\operatorname{Re}_{x} \approx 4.10 \cdot 10^{5}$ and $\operatorname{Re}_{x} \approx 4.20 \cdot 10^{5}$, respectively. The agreement between the location of $\lambda$ vortex generation and their decay into turbulence measured by TSP and hot-film anemometry is very good. It can be seen that the spanwise position is reflected by the area of laminar flow between the two wedges, which is characterized 


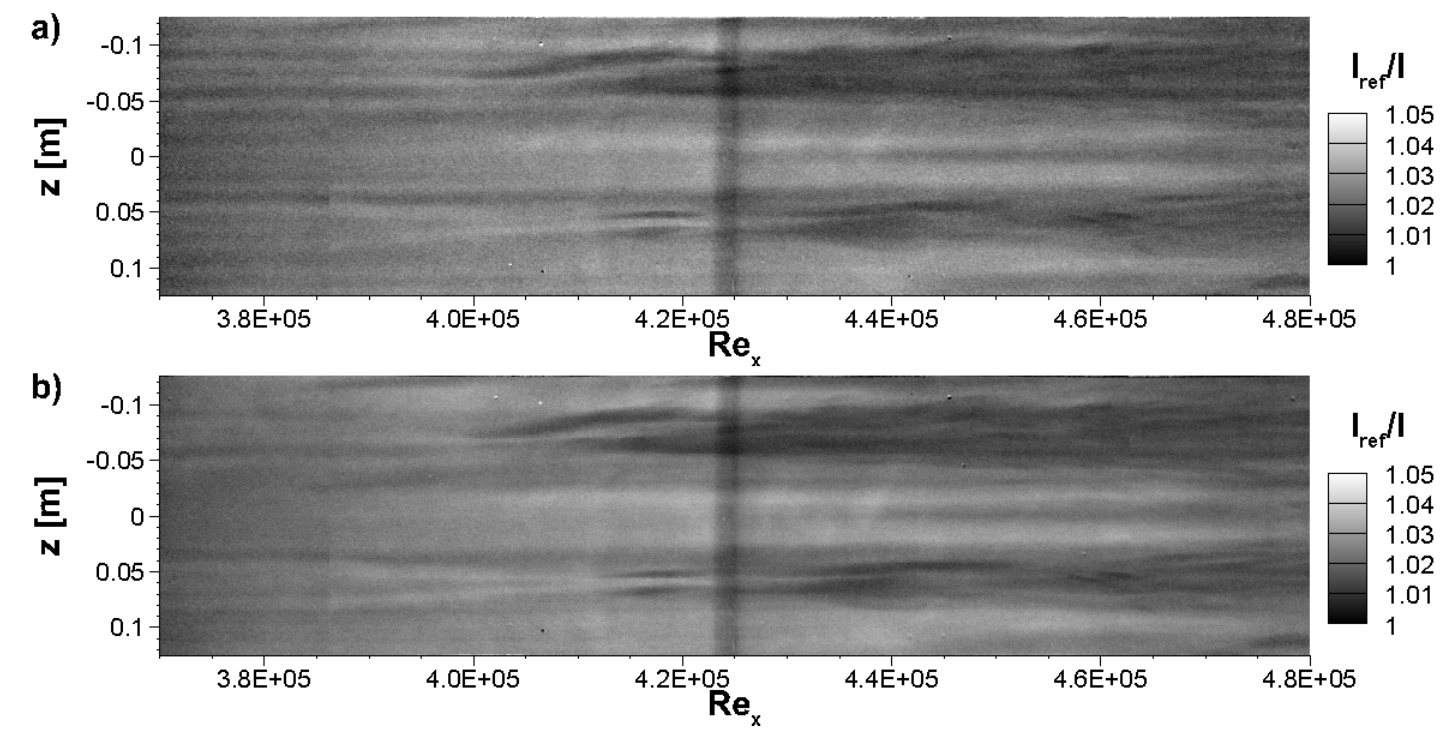

Figure 7: Instantaneous result of the reference, i.e. case (II), unfiltered a) and filtered with SVD b). The signal-to-noise ratio is increased and the horizontal stripes are successfully reduced by the filtering.

by lower flow speeds and a lower heat transfer, which is visible in Figure 6 a) and Figure 8 a), respectively.

A significant difference between the averaged TSP results and the averaged hot-film measurement is visible from the beginning of the measurement up to $\operatorname{Re}_{x} \approx 3.90 \cdot 10^{5}$. Here the results of the hot-film measurement already indicate a deformed boundary layer that is accelerated between the two growing disturbances. This is probably caused by the influence of the forward-facing steps at the beginning of the TSP element, which causes accelerated flow and improved heat transfer. In the TSP result this effect is indicated by a slightly lower intensity ratio.

\subsection{Adequately-Sized Roughness Elements}

When the roughness elements are placed $0.275 \mathrm{~m}$ away from the leading edge of the flat plate, c.f. case (IV) in Table 1, the transition is delayed beyond the trailing edge of the TSP element. For the parameter of case (IV) $k / \delta=2.27$ which is significantly smaller than the value of Fransson et al. (2005a). The roughness Reynolds number is $\operatorname{Re}_{k k}=263$ and thus smaller than for the scaled elements of case (I). This location of the roughness elements, which results in the maximum achievable transition delay, was determined experimentally. The average TSP result in Figure 9 a) shows an intensity ratio $I_{r e f} / I \geq 1.02$, which is already a strong indication for laminar flow over the entire TSP element. The lowest values are visible at the leading edge of the TSP element where the flow speed is increased also for the reference flow. Also, footprints of the roughness induced streaks inside of the laminar boundary layer are visible. At the upstream end of the TSP element these have the spacing of the roughness elements. Whereas the spacing of the streaks becomes more narrow at the downstream end. Besides the flow structures and the signature of the heating gap an additional frayed structure is visible between $\operatorname{Re}_{x} \approx 4.15 \cdot 10^{5}$ and $4.25 \cdot 10^{5}$. This structure is also expected to be caused by the model.

In the results of the TSP standard deviation (Figure 9 b)) the only area of increased values is visible at the leading edge, where the velocity is increased, and very locally at the end of the TSP element. The standard deviation increase at the downstream end shows a small spaced horizontal pattern. The amplitude spectrum (Figure 9 c)) does not show any increase of the disturbance frequency amplitude.

The results of the hot-film measurement are illustrated in Figure 10. Also for this configuration the velocity $\mathrm{u}$ is increased at the leading edge of the TSP element up to approximately $\operatorname{Re}_{x} \approx 4.00 \cdot 10^{5}$. But with the installed oblique roughness elements the induced streak system is visible over the entire length of the TSP element and the spacing of this streak system is equal to the spacing of the roughness elements $(\Delta z=11 k)$. Inside of the streaks the standard deviation $\operatorname{std}(u)$ grows, as can be seen in Figure 10 b), but does not reach the magnitude measured at transition for the reference case, c.f. Figure $8 b$ ). Both measurement techniques show that the entire TSP element is 


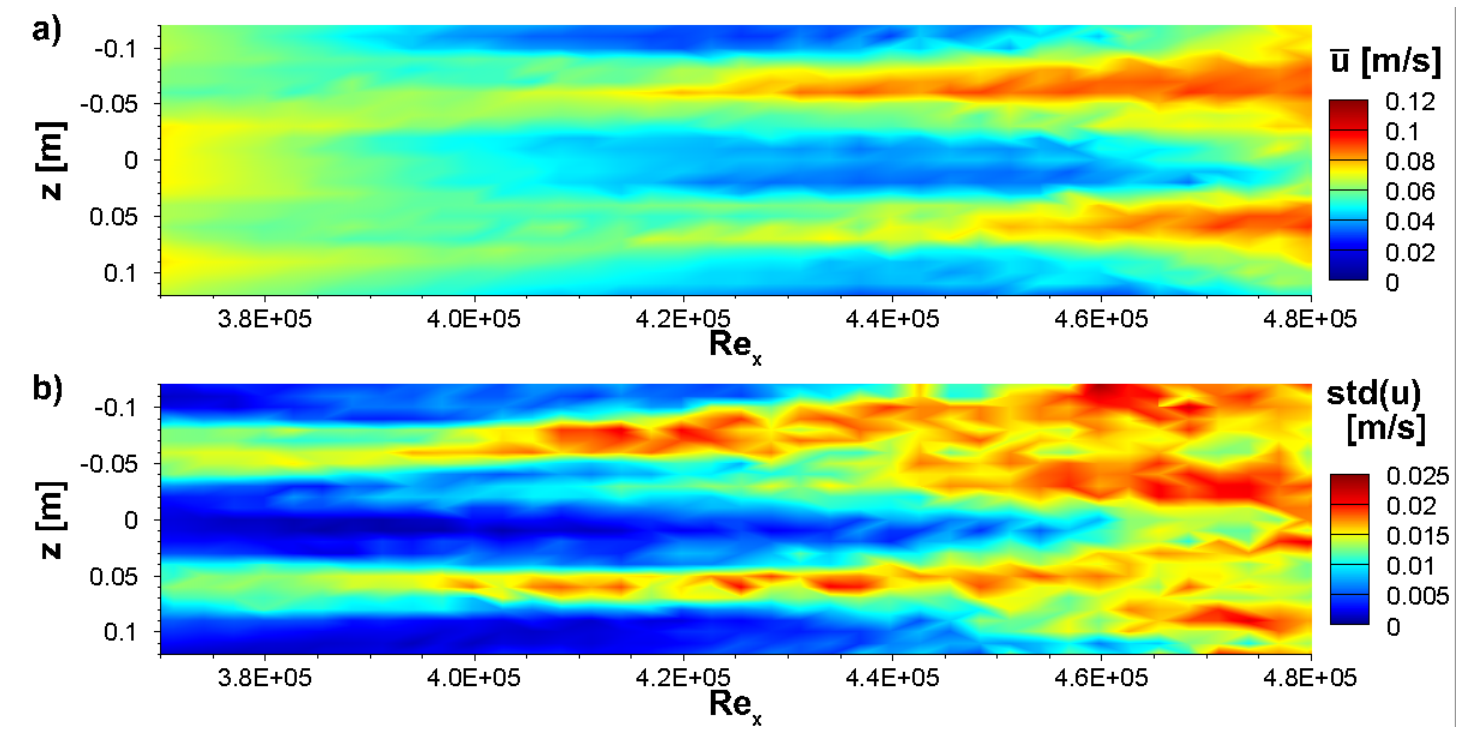

Figure 8: Hot-film results for the reference, i.e. case (II). The average velocity a) and the standard deviation of the velocity $b$ ) are shown.

now laminar. But the surface visualization does not capture the steady deformation of the boundary layer as good as the velocity measurement. As it can be concluded from the standard deviation of the velocity in Figure $10 \mathrm{~b}$ ) only the streaks with the largest standard deviation $(z / k=-11$ and $z / k=-22)$ are visible in the averaged TSP result (Figure 9 ).

\subsection{Oversized Roughness Elements}

The interpretation of the results of case (III), in which the roughness elements are placed at $x_{k}=0.225 \mathrm{~m}$, is less distinct. The roughness elements are with $k / \delta=$ 2.51 and $\operatorname{Re}_{k k}=281$ of significantly larger impact on the boundary layer than in case (IV). Therefore, streaks of larger amplitude can be expected in the boundary layer and an even later laminar-turbulent transition than for case (IV). The average result in Figure 11 a) is very similar to the average result of case (IV) in Figure 9 a). The generated high speed streaks with the roughness spacing of eleven roughness heights are visible in the upstream region of the TSP element and additionally finer spaced streaks are visible at the downstream end of the TSP element. Also, the results of the Fourier analysis of the $F=90 \pm 10$ frequency range (Figure 11 c)) just show a small increase of the amplitude in the investigated frequency range. Both results indicate that transition does not occur on the TSP element by the introduced instability, which is described in case (II).

The results of the standard deviation in Figure 11 b) and the instantaneous result in Figure 12 show that locally the laminar-turbulent transition occurs earlier and by a different instability mechanism. The roughness elements of case (III) have a roughness Reynolds number of $\operatorname{Re}_{k k}=281$. Placing the roughness elements at this location leads to an unstable wake, which is indicated by occurring turbulent spots. The combination of locally too high deformation of the boundary layer by the vortices downstream of the oblique roughness elements probably initiates the onset of the turbulent spot. This causes an already intermittent flow upstream of the location of the TSP measurements.

From the propagation of a turbulent spot an instantaneous result is illustrated in Figure 12 . The turbulent spot was initiated upstream of the TSP element and its propagation over the TSP element is clearly visible in the time-series data. The zones of a turbulent spot described by Gad-El-Hak et al. (1981) can be identified in the instantaneous result of Figure 12, Region (1) is the head of the spot, which is turbulent, detached from the wall, and overhangs region (2) which is an area of laminar fluid. Due to region (1) being detached from the surface it cannot be measured with the TSP method. What is very well captured, are regions (3) to (5). The downstream end of region (2) is characterized by the breakdown of the laminar flow and turbulence is generated. Clearly series of bursting events can be seen in the instantaneous result at the indicated border between section (2) and (3). These events gradually break up the footprint of the system of streaks that are introduced by the oblique surface roughness elements. The turbulent flow overhanging region (2) seems not 

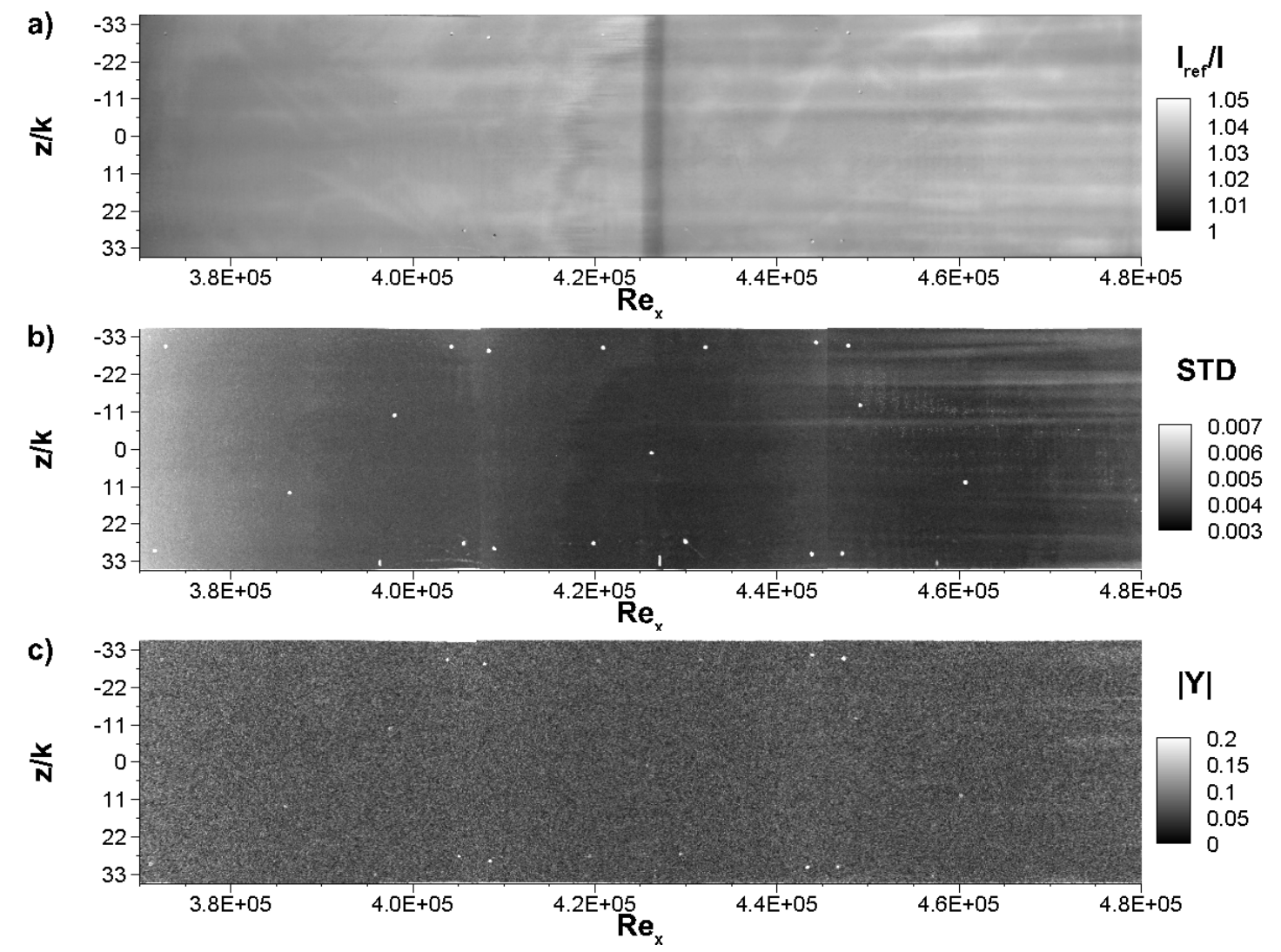

Figure 9: TSP results for successfully delayed transition with oblique roughness elements of adequate size, i.e. $k / \delta=2.27$ of case (IV). The averaged result a), the standard deviation $b$ ), and the amplitude of the $F=90 \pm 10$ frequency range derived from Fourier analysis $c$ ) are shown.

to influence the structure visible in TSP. Region (3) is characterized by very quickly growing structures that grow especially in streamwise direction. In Region (4) these structures grow further and become less active, weaken and develop into the streaks of region (5).

At last, the calmed region, i.e. region (5), follows. This region is again laminar but with the streaks embedded from the turbulent spot. After the streaks of the spots decayed completely the system of high and low speed streaks starts to develop once more if the distance between two turbulent spots is long enough. For the conditions of case (III) the distance between two consecutive turbulent spots is long enough to again form the system of high and low speed streaks induced by the roughness elements and stabilize the introduced disturbance. After a spot passed and the calmed region has passed the TSP element, it takes less than 20 seconds to reinstall the system of roughness induced streaks. The footprint of the streak can be observed growing in downstream direction.

The footprint of the stabilizing streak is clearly visible in the average result, standard deviation, and amplitude spectrum (top right corner of Figure 11) because the turbulent spot did not overflow the entire TSP element during the acquisition of the data point. The undisturbed areas show a stabilized boundary layer as in Figure 9 for roughness elements with smaller impact on the boundary layer, i.e. the generated streaks are weaker. The measurement time of this intermittent flow was insufficient to capture the flows characteristics completely.

The turbulent spots seem not to be randomly initiated in spanwise direction because most spots passing the area of TSP visualization were initiated on the positive z-side of the test section. Thus, there seems to be a defect of a roughness element, e.g. inaccurate orientation.

As expected for an off-design condition with oversized oblique roughness elements the wake of the roughness elements becomes unstable and the transition location moves rapidly forward. The investigated roughness shape becomes already unstable from $\operatorname{Re}_{k k} \approx 280$ on. 


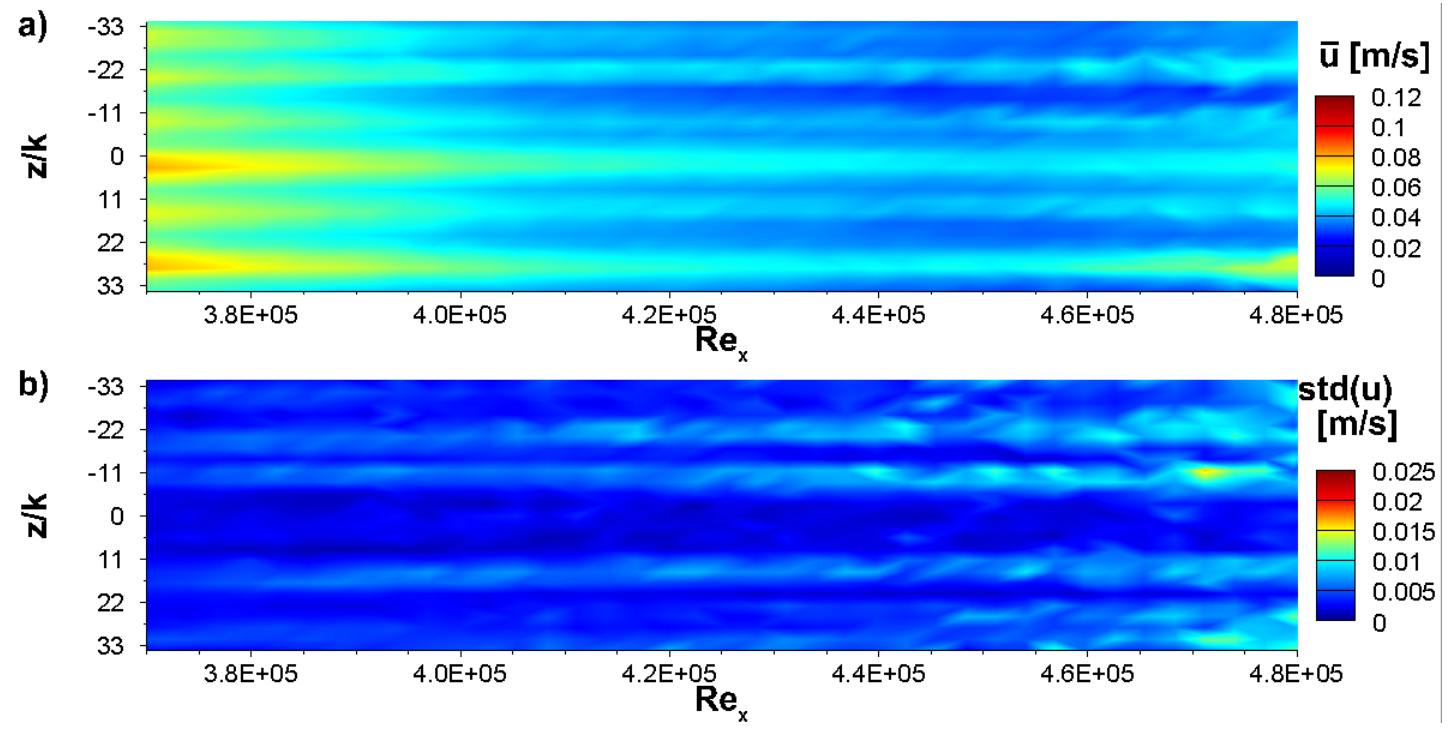

Figure 10: Hot-film results for the transition delayed with roughness elements of adequate size, i.e. case (IV). The average velocity a) and the standard deviation of the velocity $b$ ) is shown.

\subsection{Insufficiently Sized Roughness Ele- indication for laminar-turbulent transition is visible. ments}

The roughness elements of case $(\mathrm{V})$ are placed further downstream then in the optimal configuration of case (IV). The roughness location yields in a roughness Reynolds number of $\operatorname{Re}_{k k}=254$ and a ratio of roughness height to boundary layer parameter of $k / \delta=2.09$. The average result is shown in Figure 13 a) and is similar to the average result of the reference flow in Figure 6 a). The steady deformation of the boundary layer leads to a structure of two thin horizontal lines on the negative $z$ side of increased heat transfer that become wider downstream of the gap in the heating. This structure has the width of the spacers beneath the vibrating ribbon. On the positive $z$-axis the structure appears later than on the negative $z$-axis. But the overall beginning of the structure has moved further downstream with a more pronounced asymmetry between positive and negative $\mathrm{z}$ coordinates than in the reference flow.

The standard deviation and amplitude spectrum results are shown in Figure 13 b) and c), respectively. These results show an elongated, smeared out maximum of lower amplitude than for the reference flow. This is an indication for a less steady location of production of the $\lambda$ vortices or a less regular production. The maximum in the standard deviation and amplitude spectrum is located further downstream than in the reference case and transition is therefore expected to be still delayed. On the positive $z$ coordinate side no
In the average result no turbulent area is visible but lambda vortices are still initiated. The initiation and the growth of the lambda vortices are visible in the instantaneous images (not shown). It is also visible that they can become unstable and decay into turbulent flow within the range of the TSP element but this occurs not as regular as for the reference flow condition. This behavior is reflected by an area with increased amplitude of the $F=90 \pm 10$ frequency band but no indication of turbulent flow in the average result. The increased amplitude of the $F=90 \pm 10$ frequency band is limited to the initial width of the footprint of the lambda vortices in its spanwise extent and does not increase in downstream direction. But this area of increased disturbance is elongated in flow direction compared to the reference flow condition. Hence, the stabilizing effect of the oblique surface elements is visible because the disturbance reaches sufficient strength to be visible in the TSP results further downstream than in reference flow and the average distance to decay into turbulent flow is also elongated. This area of increased amplitude in the $F=90 \pm 10$ frequency band moves gradually in upstream direction when $k / \delta$ is further decreased. This off-design behavior of the oblique roughness elements is less critical: With decreasing influence of the streaks the transition is less delayed. 

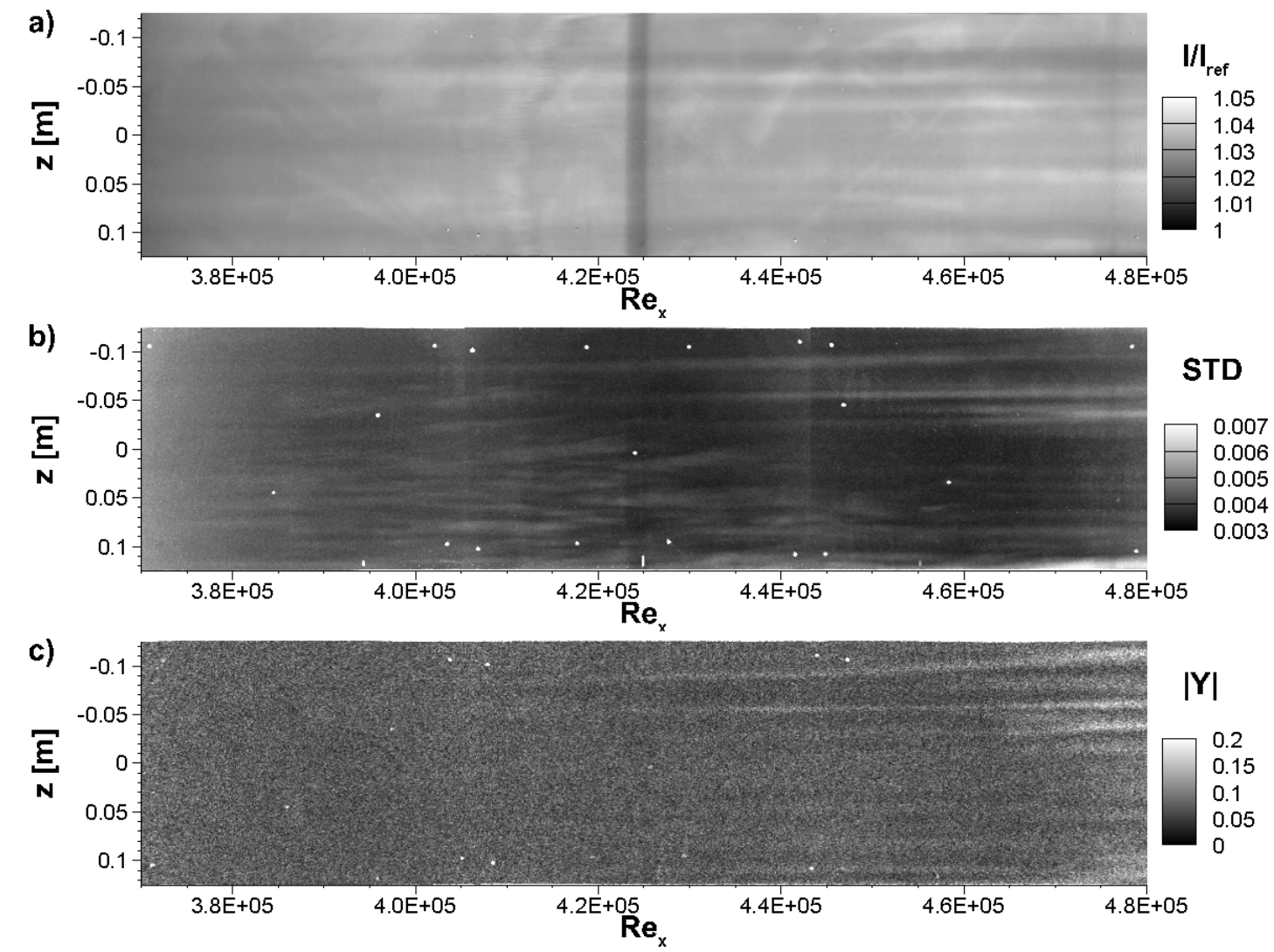

Figure 11: TSP results for roughness elements with $k / \delta=2.51$ and $R e_{k k}=281$, i.e. case (III). The averaged result a), the standard deviation $b$ ), and the amplitude of the $F=90 \pm 10$ frequency range derived from Fourier analysis $c$ ) are shown.

\section{Conclusions}

In the present investigation the TSP method was successfully used to spatially and temporarily measure a triggered K-type (Kruse and Wagner, 1998; Klebanoff et al. 1962) laminar-turbulent transition of a Blasius flow. To enable TSP measurements in the laminar water channel a TSP element was designed, which can be easily relocated on the flat plate of the laminar water channel. Surface visualizations with TSP are in good agreement with velocity measurement results from hot-film anemometry measurements for K-type transition. In the hot-film measurements a flow alteration at the beginning of the TSP element is clearly detected. This flow alteration is caused by the forward-facing step of the TSP element.

The laminar-turbulent transition of the Blasius boundary-layer flow with K-type transition was successfully delayed by placing oblique surface roughness elements close to the leading edge of the flat plate. The cuboid elements placed under $45^{\circ}$ to the oncoming flow generate a complex vortex system very similar to the numerical results of Groskopf et al.
(2011). Only the vortex generated at the leading edge of the cuboid persists. These form a system of corotating vortices, which generate high and low speed streaks. The streaks deform the Tollmien-Schlichting waves into three-dimensional waves with a reduced growth rate. With the roughness elements beneficially placed at $k / \delta=2.27$ with a spanwise spacing of $k / z=11$ (case (IV)) the transition was delayed by at least $\Delta \operatorname{Re}_{x}=80.000$ in the present experiment. The exact transition location could not be determined due to an insufficient size of the TSP element. In previous experimental work pairs of counter-rotating vortices were utilized (Fransson et al. 2005a: Shahinfar et al. 2012). The roughness elements in the presented investigation are smaller and have a larger spanwise spacing than in the previous works; however, transition was successfully delayed.

Also, the off-design conditions, i.e. oversized (case (III)) and insufficiently sized (case (V)) roughness elements, were tested. The wake of the roughness elements becomes unstable from $\operatorname{Re}_{k k}=282$ on and is fully turbulent from $\operatorname{Re}_{k k}=307$ on. The unstable flow is characterized by occurring turbulent spots, which 


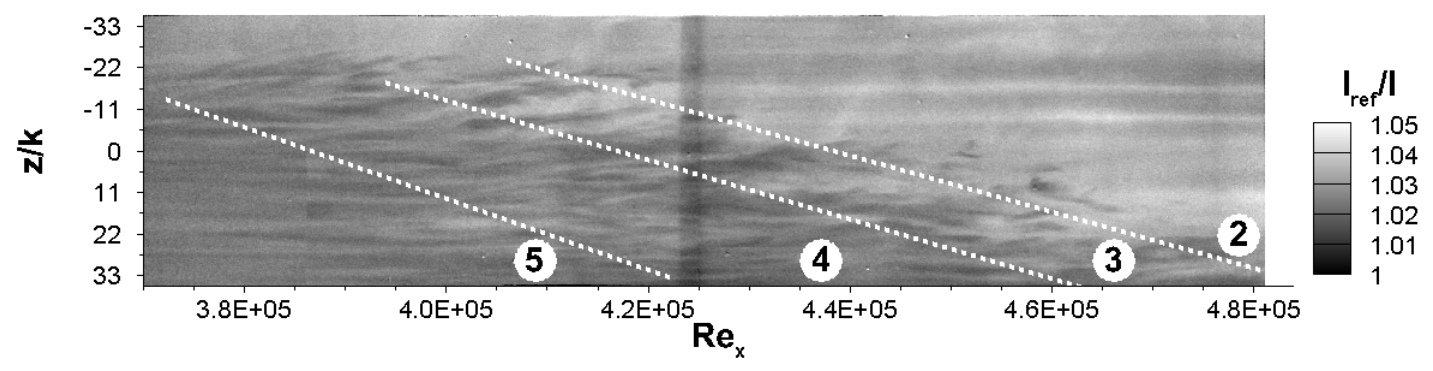

Figure 12: TSP instantaneous visualization result for roughness elements with $\operatorname{Re}_{k k}=281$, i.e. case (III). The different regions of the visible turbulent spot are indicated and denoted following the notation of Gad-El-Hak et al. (1981).

disturb the system of stabilizing streaks. If the roughness elements are insufficiently sized, the TollmienSchlichting waves are insufficiently stabilized and the transition location moves again closer to the transition location of the reference flow.

The TSP method captured the changes of the transition location and the differences in the transition process well. Furthermore, the TSP method directly allows a qualitative interpretation of the change in the surface temperature to a changed skin friction drag: The percentage of the TSP elements surface with an intensity ratio of $I_{r e f} / I \geq 1.025$ is significantly increased when the roughness elements are added (case (IV)). Especially, the highly unsteady and three-dimensional flow with turbulent spots (case (III)) can be well investigated with the TSP method, because of its spatial and temporal measurement capabilities. To further improve measurement results in future investigations the TSP element should be flush mounted with the surface of the already installed flat plate, i.e. an existing part of the flat plate should be replaced with a TSP element.

Acknowledgements The authors would like to thank Mr. Carsten Fuchs (DLR-AS-EXV), Mr Tobias Kleindienst (DLR-AS-EXV), Dr. Vladimir Ondrus (University of Hohenheim), Ms. Esther Mäteling (RWTH Aachen), and Mr. Martin Weberschock (Weberschock Development) for their support during the design, manufacturing, and preparation of the TSP element.

\section{REFERENCES}

C. Cossu and L. Brandt. On tollmien-schlichting-like waves in streaky boundary layers. EUR J MECH B-Fluid, 23: 815-833, 2004. doi: 10.1016/j.euromechflu.2004.05.001.

R. S. Downs III and J. H. M. Fransson. Tollmien-Schlichting wave growth over spanwise-periodic surface patterns. J.
Fluid Mech., 754:39-74, 2014. doi: doi:10.1017/jfm.2014. 377.

U. Fey and Y. Egami. Springer Handbook of Experimental Fluid Mechanics, chapter Transition-Detection by TemperatureSensitive Paint, pages 537-552. Springer-Verlag, Berlin, Heidelberg, New York, 2007.

U. Fey, K. de Groot, and Y. Le Sant. Thermography as a tool in wind tunnel testing. Technical Report DLR-IB 224-2007 A 09, German Aerospace Center (DLR), 2007.

U. Fey, C. Klein, T.J. Möller, J. Pöttner, R. Radespiel, V. Ondrus, and U. Beifuß. Notes on Numerical Fluid Mechanics and Multidisziplinary Design, volume 121, chapter Investigation of Circular Cylinder Flow in Water Using Temperature-Sensitive Paint, pages 657-664. SpringerVerlag, 2013.

J. H. M. Fransson, L. Brandt, A. Talamelli, and C. Cossu. Experimental study of the stabilization of TollmienSchlichting waves by finite amplitude streaks. Phys. Fluids, 17(054110):10, 2005a. doi: doi.org/10.1063/1.1897377.

J. H. M. Fransson, M. Matsubara, and P.H. Alfredsson. Transition induced by free-stream turbulence. J. Fluid Mech., 527:1-25, 2005b. doi: doi.org/10.1017/ S0022112004002770.

M. Gad-El-Hak, R. F. Blackwelder, and J. J. Riley. On the growth of turbulent regions in laminar boundary layers. J Fluid Mech, 110:73-95, 1981.

G. Groskopf, M. J. Kloker, and K. A. Stephani. Temperature / rarefaction effects in hypersonic boundary-layer flow with an oblique roughness element. In 41st AIAA Fluid Dynamics Conference and Exhibit, 27 - 30 June 2011, Honolulu, Hawaii, number AIAA 2011-3251, 2011.

F.R. Hama and J. Nutant. Detailed flow-field observations in the transition process in a thick boundary layer. In Proceedings of the 1963 Heat Transfer and Fluid Mechanics Institute, Stanford University Press, 1963.

P. S. Klebanoff, K. D. Tidstrom, and L. M. Sargent. The three-dimensional nature of boundary-layer instability. $J$. Fluid Mech., 12(1):1-34, 1962. 

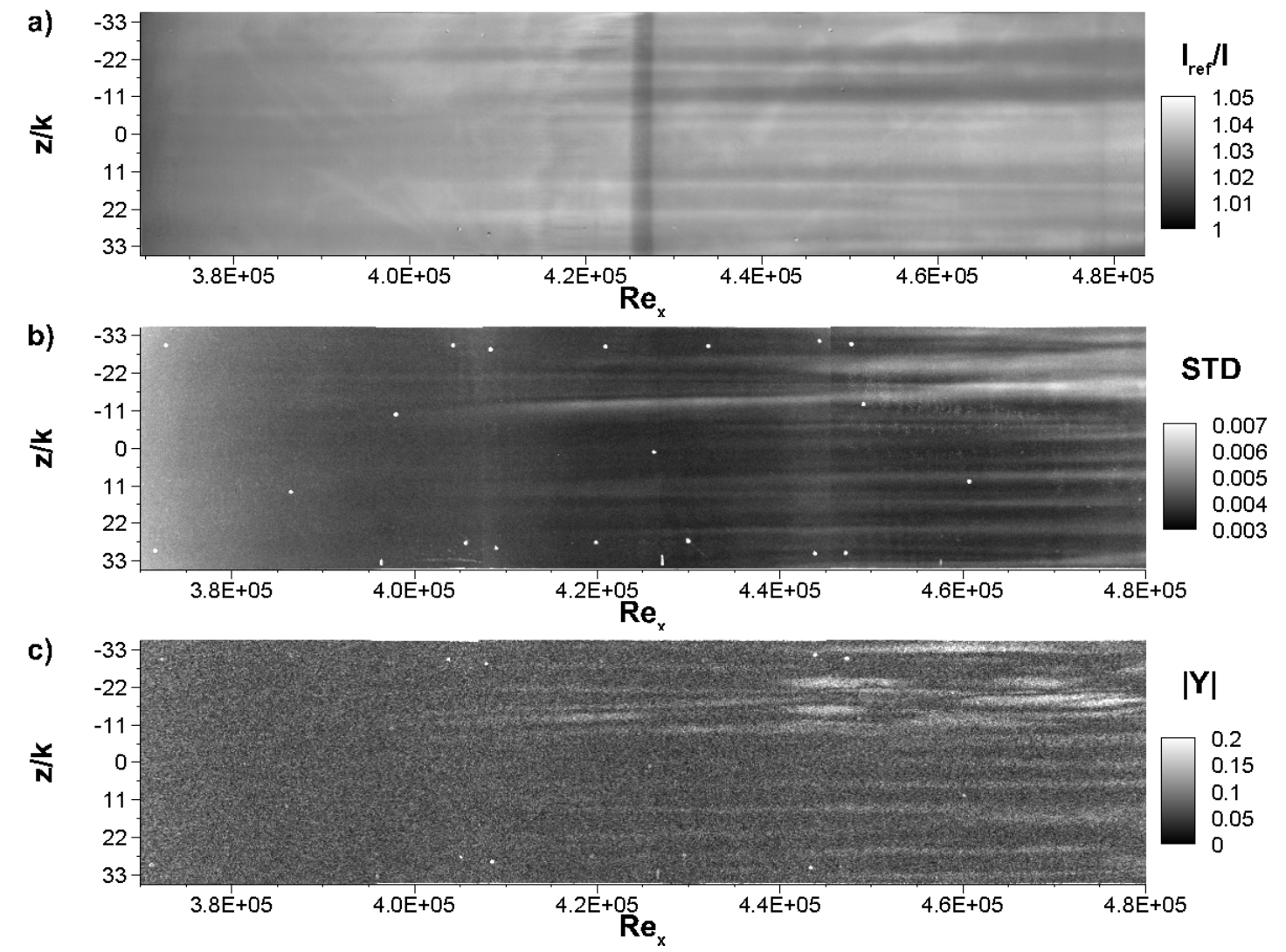

Figure 13: TSP results for insufficiently sized roughness elements with $k / \delta=2.09$ and $\operatorname{Re}_{k k}=254$, i.e. case $(V)$. The averaged result a), the standard deviation $b$ ), and the amplitude of the $F=90 \pm 10$ frequency range derived from Fourier analysis $c$ ) are shown.

K. Konstantinides, B.K. Natarajan, and G.S. Yovanof. Noise estimation and filtering usoing block-based singular value decomposition. IEEE Transactions on Image Processing, 6 (3):479-483, 1997.

M. Kruse and S. Wagner. Visualization and laser Doppler measurements of the development of Lambda vortices in laminar-turbulent transition. Meas. Sci. Technol., 9(4): 659-669, 1998.

M. T. Landahl. A note on an algebraic instability of inviscid parallel shear flows. J. Fluid Mech., 98:243-251, 1980.

J. Lemarechal, E. Mäteling, C. Klein, D. Puckert, and U. Rist. Reattaching flow behind a forward-backward facing step investigated with temperature sensitive paint. In A. Dillmann, G. Heller, E. Krämer, C. Wagner, S. Bansmer, R. Radespiel, and R. Semaan, editors, New Results in Numerical and Experimental Fluid Mechanics XI, pages 285-294. Springer International Publishing, 2018.

T. Liu and J. P. Sullivan. Pressure And Temperature Sensitive Paints. Springer-Verlag, 2005.

K. Nakakita. Unsteady pressure distribution measurement around 2D-cylinders using pressure-sensitive paint. In 25th AIAA Applied Aerodynamics Conference, 25 - 28 June 2007, Miami, FL, number AIAA 2007-3819, 2007.

V. Ondrus, R. J. Meier, C. Klein, U. Henne, M. Schäferling, and U. Beifuß. Europium 1,3-di(thienyl)propane1,3-diones with outstanding properties for temperature sensing. Sensors and Actuators, A: Physical, 233:434-441, 2015.

M. Pastuhoff, D. Yorita, K. Asai, and P.H. Alfredsson. Enhancing the signal-to-moise ratio of pressure sensitive paint data by singular value decomposition. Meas. Sci. Technol., 24(7):075301, 2013. doi: doi:10.1088/0957-0233/ 24/7/075301.

R. Petzold and R. Radespiel. Transition on a wing with spanwise varying crossflow and linear stability analysis. AIAA Journal, 53(2):321-335, 2015.

J. Reneaux. Overview on drag reduction technologies for civil transport aircraft. In European Congress on Computational Methods in Applied Sciences and Engineering ECCOMAS 2004, University Jyväskylä, Finland, 2004.

W.E. Sachs. Topas source code, design principles, basic source code, $2 \mathrm{~d}$ source code, $3 \mathrm{~d}$ source code. Technical 
Report DLR-IB-2007 C22-24, German Aerospace Center (DLR), 2007.

G. Schrauf. Status and perspectives of laminar flow. The Aeronautical Journal, 109(1102):639-644, 2005.

S. Shahinfar, S.S. Sattarzadeh, J.H.M. Fransson, L. Brandt, A. Talamelli, and C. Cossu. Revival of classical vortex generators now for transition delay. PHYSICAL REVIEW LETTERS, 109, 2012. doi: doi.org/10.1103/PhysRevLett. 109.074501 .

M. Strunz. Ein Laminarwasserkanal zur Untersuchung von Stabilitätsproblemen in der Strömungsgrenzschicht. PhD thesis, Universität Stuttgart, 1987.

A Subasi, D. Puckert, H. Gunes, and U. Rist. Calibration of constant temperature anemometry with hot-film probes for low speed laminar water channel flows. In 13th International Symposium on Fluid Control, Measurement and Visualization FLUCOME2015, 15-18 November, Doha, Qatar, 2015.

K. Taira, S.L. Brunton, S.T.M. Dawson, C.W. Rowley, T. Colonius, B.J. McKeon, O.T. Schmidt, S. Gordeyev, V. Theofilis, and L.S. Ukeiley. Modal analysis of fluid flows: An overview. AIAA Journal, 55(12):4013-4041, 2017.

L. Wang. Measuring Optical Absorption Coefficient of Pure Water in UV Using the Integrating Cavity Absorption Meter. PhD thesis, Texas A\&M University, 2008. 